\title{
Overcrowding and "floor" patients in state hospitals: institutions can make a difference
}

\author{
C Delpachithra' and Saroj Jayasinghe ${ }^{2}$
}

\section{Rationalising admissions to hospital, improving outpatient and ambulatory care services, improved bed utilisation and increasing turnover rate are likely to make a significant difference}

Two recent articles and one official document have drawn attention to the problem and implications of overcrowding in state hospitals, especially the issues related to floor patients $(1,2,3)$. The proposals to counter overcrowding include those based on the reasons for overcrowding at tertiary care level, bypassing of other levels of care, lack of an effective referral system, and the increasing demand for specialist care (3). The long term strategies aimed at the level of the health system appear to have had little impact on the situation. If at all, in the recent past (1994 to 1998), the occupancy rates have increased in tertiary care hospitals, in contrast to other levels of care (Table 1). The reason may be slow implementation of these strategies, because that requires heavy investment of resources (eg. improving services in other levels of care), and the controversial nature of some (eg. delinking primary care from tertiary care hospitals).

Table 1. Occupancy rates and duration of stay in state hospitals 1994 and $1998(4,5)$

\begin{tabular}{lcccc} 
& \multicolumn{2}{c}{$\begin{array}{c}\text { Bed occupancy rates } \\
\text { (\%) }\end{array}$} & \multicolumn{2}{c}{$\begin{array}{c}\text { Average duration of } \\
\text { stay (days) }\end{array}$} \\
& 1994 & 1998 & 1994 & 1998 \\
$\begin{array}{l}\text { National } \\
\text { Hospital, } \\
\text { Colombo* }\end{array}$ & 99 & 104.7 & 5.7 & 5.7 \\
$\begin{array}{l}\text { Other Teaching } \\
\text { Hospitals* }\end{array}$ & 91 & 91.3 & 5.1 & 5.2 \\
$\begin{array}{l}\text { Provincial } \\
\text { Hospitals* }\end{array}$ & 103 & 106 & 4.9 & 4.7 \\
$\begin{array}{l}\text { Base Hospitals } \\
\text { Mistrict }\end{array}$ & 91 & 85.7 & 4.2 & 3.5 \\
Hospitals & 59 & 51.6 & 3.1 & 2.6 \\
* Could be considered as tertiary care facilities &
\end{tabular}

Establishing a referral system is probably not going to be effective for a number of reasons. First, most medical officers in the state sector enjoy the privilege of private practice. The obvious mechanism to bypass a rigid referral system would be to consult the state sector medical officers in their private practice and "jump the queue". Secondly, establishing a referral system would require more resources in an already resource scarce health sector. To start with the simplest, how are we going to have a forgery proof referral form? Can we prevent duplicate versions of the referral forms being printed and sold at a price? Can we prevent errant medical officers taking referral forms to their private practice? Thirdly, the UK system is used as an example of successful implementation of a referral system, without realising how different the UK system is from Sri Lanka. Their GPs are linked to the state sector, medical records are excellent, and trusts with responsibility to use funds have taken over ownership of certain institutions. In Sri Lanka, there is hardly any regulation of private practice, a medical records system is almost non-existent and the hospitals are under two administrative structures (ie. teaching hospitals under the "centre", and the other institutions under the Provinces).

In addition to long term and system-wide strategies, there are other interventions which should reduce overcrowding. They include institutional level interventions and improvement of ambulatory care services. These appear to have received scant attention by health policy planners. The present article proposes a few for discussion and debate. At the level of the institution, strategies to reduce overcowding are to rationalise admissions (admit only the deserving), use all available beds within the hospital, and increase rate of turnover.

\section{Rationalise admissions}

State hospitals have a policy of not denying admissions to the needy. We firmly believe that this policy should not be changed for two reasons: poverty in rampant in Sri Lanka and costs of private health care are increasing, making private health care unaffordable to many. Proposals to rationalise admissions include de-linking primary care facilities from tertiary care hospitals and confining out-patient services (OPDs) in tertiary centres to referral clinics (3). Such proposals are barriers to patients, and are

'Intern medical officer and ${ }^{2}$ Associate Professor, Department of Clinical Medicine, Faculty of Medicine, University of Colombo. (Accepted 31 March 2001). 
likely to increase inequities and corruption. Linking of several institutions to provide beds is not feasible, because of the complex organisational changes required for implementation (eg. agreement between hospitals, lack of communication and ambulance facilities).

Can we improve the efficiency of existing OPDs to promote more rational admissions? Two strategies are proposed: improve the services available at OPDs and improve the performance of OPD medical officers.

\section{Improve services available at OPDs}

Organisational changes which may improve the functioning of OPDs include, more autonomy in financing and management (eg. a separate budget and directorate), more resources (eg. adequate stationery, improved infrastructure, and basic 24-hour laboratory, pharmacy and radiological services), and improved record keeping. For example a 24-hour pharmacy linked to the OPD would enable patients to get the necessary drugs, rather than having to be admitted, especially at night. A 24-hour radiological service would enable many respiratory problems or those related to trauma to be sorted out within minutes.

As regards improving services, we present data that support the setting up of "preliminary care units" (PCUs), an idea proposed by Dr Dhanapala Rodrigo, an anaesthetist now domiciled in the US. Briefly, a PCU is an observation ward for all potential admissions to the hospital, with emergency care being given by OPD medical officers. Decisions to admit for further in-patient care, referral to clinics and discharge are made by the "on call" team who visit the PCU. In September 2000 we obtained prospective data from the National Hospital Colombo (NHC), on the numbers attending the OPD, numbers admitted to the wards, and outcomes. The OPD of the NHC caters to about 1500 patients each working day, of whom about 450 (28.9\%) are admitted to the hospital, excluding accident service admissions. Almost $20 \%$ of admissions occurred between 08.00 and 11.30 hours. We followed up a cohort of a random sample of patients $(n=48)$ admitted over 5 consecutive weekdays admitted to the general medical and surgical wards. Admissions to the accident service, special units, and internal transfers were excluded. Of the cohort, $20.8 \%$ were discharged within 24 hours and 50\% within 48 hours. This means that an effective PCU with appropriate laboratory and pharmacy facilities should reduce the admissions to NHC general medical and surgical wards by $20 \%$, eliminate or greatly reduce the "floor" patient population at $\mathrm{NHC}$, and also save money.

To be effective, the PCU at the NHC should be large (the conventional 60 beds would be inadequate), close to the OPD, and have surgical and medical casualty wards in one location to facilitate cross referrals. Presently, the casualty ward system at the NHC is disjointed. The medical (male and female) and surgical (male) casualty wards are situated away from the OPD, and located in separate buildings. They have a combined bed strength of 68 , and there is no separate surgical casualty ward for females.

\section{Improve the performance of OPD medical officers}

There is room to improve the performance of OPD medical officers, as there is a hiatus in their training at both undergraduate and postgraduate levels. The place of ambulatory care (which includes first contact care, primary health care, follow-up care after an illness episode and care of chronically ill) in medical education and in human resource deployment in Sri Lanka is most inadequate. Even within the specialty of ambulatory care, the emphasis is not on learning about the common "episodic" consultation, ie. a consultation for a particular illness or health related problem. This is in contrast to the 3 models emphasised in medical education.

a) British model of General Practice (GP with links to the state sector and follow up notes)

b) The Family Physician (FP) model (with independent or groups of private practitioners with follow up facilities) and

(c) The Medical Officer of Health (MOH) model, practiced in the preventive services of Sri Lanka.

Policy makers have several options in this regard: to move towards a preferred form of care (ie. GP, FP or MOH) and to improve the episodic care facilities in our hospitals. To do either or both of these, we should improve the ambulatory care services in our hospitals and improve the quantum and quality of staff at OPDs.

The following proposals should be considered as a matter of urgency:

i) Use a proportion of the large influx of doctors now overcrowding the curative sector to man OPDs. They should be given an orientation in ambulatory care (especially "episodic" care). With increase in the number of doctors available, a more effective records system should be introduced with a system for follow up, so that a patient is able to see a particular doctor on a continuing basis, at least for a few visits.

ii) Improve the training of medical students on providing ambulatory care. Unfortunately in Sri Lanka we continue to downgrade the importance of ambulatory care and almost totally ignore training in providing "episodic" care. At a time when ambulatory care is becoming a specialty in its own right, we appear to be going in the opposite direction, with a heavier bias towards curative tertiary care. For example, the Faculty of Medicine in Colombo is right now debating whether programs in primary care facilities introduced in 1995 (which consists of only about 10\% of clinical appointments), should be pruned further.

iii) Improve the training modules in the postgraduate training programs for medical administrators on development and administration of ambulatory care services. Training in development of ambulatory care services is often diluted by the emphasis given to the hospital administration and preventive services. The College of Medical Administrators could take a lead in this area. 


\section{Utilise all available beds within the hospital}

Why don't private hospitals (which too are under pressures to admit), have "floor" patients? There are two likely reasons. First, private hospitals refuse admission if there are no beds. Secondly, private sector hospitals utilise beds to a maximum by having wards with a mix of patients, unlike the unit system in state hospitals. The latter enables an admission to be accepted if a bed is vacant anywhere in the hospital. To implement this system, we have to agree to do away with strictly demarcated wards dedicated to a specialist or to certain specialties, a practice in many countries where most of our specialists train. It is also necessary to have a special unit to coordinate admissions, smaller wards with adequate numbers of nursing and other staff and basic equipment. Such a system could be implemented in a limited manner, at least in the newer hospitals being constructed in the country. The guiding principle should be not to have "floor" patients at least till all the beds in the hospital except those reserved for emergencies are occupied.

\section{Increase rate of turnover}

To release more vacant beds for occupation, we could increase the rate of turnover. Clinicians would agree about the delays in discharging patients because biochemistry reports or xrays are not available. This is partly a result of the rigid systems we have in our hospitals. A few examples are,

a) patients admitted after 9 a.m. have to stay overnight to have tests done the next morning. Providing a 24-hour laboratory service and radiological services for a few selected tests would enable quick decisions to be made and early treatment and discharge of patients.

b) team care of patients, with several specialists caring for a particular patient is rarely practiced even in the pri- vate sector. In our system, patients or patients' notes move around the hospital in search of specialists, rather than the doctors meeting together to see the patients. Certain specialists insist on specialist to specialist referrals. This also leads to delays. Of course, part of the reason for a poor team effort among specialists is that they are too busy because of overcrowding in the wards. Yet another reason is reluctance to put our minds together and sort out problems.

\section{Conclusions}

Having patients on the floor is an intolerable situation that denies basic human dignity to a sick human being. Though the problem may appear overwhelming, there are several options to solve the crisis at an institutional level. The need of the hour is strong commitment, willingness to innovate and a little more resources.

\section{Acknowledgement}

Part of the work was funded hy the Health Research and Development Program of the University of Colombo.

\section{References}

1. Goonawardena SAS. Towards achieving civilised status for state hospitals. Ceylon Medical Journal 2000; $45: 151$.

2. Corea E. There are people on the floor. Sri Lankan Family Physician 1998; 21: 31-2.

3. Report of the Presidential Task Force on Formulation of a National Health Policy for Sri Lanka, Sessional paper No II1993, The Department of Government Printing 1992.

4. Annual Health Bulletin. Ministry of Health Sri Lanka, 1994.

5. Annual Health Bulletin. Ministry of Health Sri Lanka, 1998. 